\title{
Fisher Vector Faces in the Wild
}

Karen Simonyan

karen@robots.ox.ac.uk

Omkar M. Parkhi

omkar@robots.ox.ac.uk

Andrea Vedaldi

vedaldi@robots.ox.ac.uk

Andrew Zisserman

az@robots.ox.ac.uk

\author{
Visual Geometry Group \\ Department of Engineering Science \\ University of Oxford
}

\begin{abstract}
Several recent papers on automatic face verification have significantly raised the performance bar by developing novel, specialised representations that outperform standard features such as SIFT for this problem.

This paper makes two contributions: first, and somewhat surprisingly, we show that Fisher vectors on densely sampled SIFT features, i.e. an off-the-shelf object recognition representation, are capable of achieving state-of-the-art face verification performance on the challenging "Labeled Faces in the Wild" benchmark; second, since Fisher vectors are very high dimensional, we show that a compact descriptor can be learnt from them using discriminative metric learning. This compact descriptor has a better recognition accuracy and is very well suited to large scale identification tasks.
\end{abstract}

\section{Introduction}

Face identification, i.e. the problem of inferring the identity of people from pictures of their face, is a key area of research in image understanding. Beyond its scientific interest, this problem has numerous and important applications in surveillance, access control, and search. Automatic Face Verification (AFV) is a formulation of the face identification problem where the task is to determine whether two images depict the same person or not. In the past few years, the dataset "Labeled Faces in the Wild" (LFW) [ $\square]$ has become the de-facto evaluation benchmark for AFV, promoting the rapid development of new and significantly improved AFV methods. Recent efforts, in particular, have focused on developing new image representations and combination of features specific to AFV to surpass standard representations such as SIFT $[\mathbb{}(\mathbb{Z}]$. The question that this paper addresses is what happens if, instead of developing yet another face-specific image representation, one applies off-the-shelf object recognition representations to AFV.

The results are striking. Our first contribution is to show that dense descriptor sampling combined with the improved Fisher Vector (FV) encoding of [四] (Sect. 2) outperforms or performs just as well as the best face verification representations, including the ones that use elaborate face landmark detectors $[\mathbf{Q}, \mathbf{\theta}]$ and multiple features $[\mathbb{\square}]$. The significance of this 
result is that FVs are not specific to faces, having been proposed for object recognition in general. However, FV descriptors are high-dimensional, which may be impractical in combination with huge face databases. Our second contribution is to show that FV face representations are amenable to discriminative dimensionality reduction using a linear projection, which leads simultaneously to a significant dimensionality reduction as well as improved recognition accuracy (Sect. 3). The processing pipeline (Sect. 4) is illustrated in Fig. 1. Our end result is a compact discriminative descriptor for face images that achieves state-of-theart performance on the challenging LFW dataset in both restricted and unrestricted settings (Sect. 5).

\subsection{Related work}

Face identification approaches. Face recognition research has been focusing on five areas: face detection, facial landmark detection, face registration, face description, and statistical learning. A typical face recognition system requires all these steps, but many works focus on a few of these aspects in order to improve the overall system performance. For facial landmark detection, Everingham et al. [ $[\mathrm{Q}]$ proposed pictorial structures, Dantone et al. [ $[\mathrm{Q}]$ conditional random forests, and Zhu et al. [Q] deformable parts models. Several papers

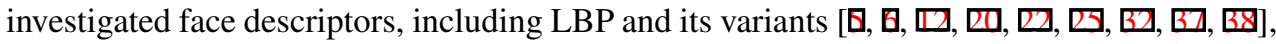

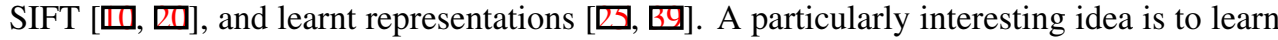
and extract semantic face attributes as facial features for identification and other tasks $[\square, \square]$. Statistical learning is generally used to map face representations to a final recognition result, with metric or similarity learning being the most popular approach, particularly for

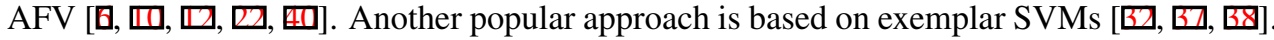

Dense features and their encodings for generic object recognition. Dense feature extraction is an essential component of many state-of-the-art image classification methods [एष, [2, $\square]$. The idea is to compute features such as SIFT densely on an image, rather than on a sparse and potentially unreliable set of points obtained from an interest point detector. Dense features are then encoded into a single feature vector, summarising the image content in a form suitable for learning and recognition. The best known encoding is probably the Bag-of-Visual-Words (BoVW) model [ [, , $]$ ], which builds a histogram of occurrences of vector-quantised descriptors. More recent encodings include VLAD [ $\square$ ], Fisher Vectors (FVs) [四], and Super Vector Coding [ㅁ] ]. A common aim of these encodings is to reduce the loss of information introduced by the vector quantisation step in BoVW. In [ [0] it was shown that FVs outperform the other encodings on a number of image recognition benchmarks, so we adopt them here for face description.

Discriminative dimensionality reduction. The aim of discriminative dimensionality reduction is to obtain smaller image descriptors, while preserving or even improving their ability to discriminate images based on their content. This is often formalised as the problem of finding a low-rank linear projection $W$ of the descriptors that minimises the distances between images with the same content (e.g. same face) and maximises it otherwise. "Fisherfaces" $[\mathbb{[}]$ is one of the early examples of discriminative learning for dimensionality reduction, applied to face recognition. A closely related formulation is that of learning a Mahalanobis matrix $M=W^{\top} W$, a problem that has convex formulations [毘, even in the case of low-rank constraints [四]. However, learning the matrix $M$ is practical only if the starting 


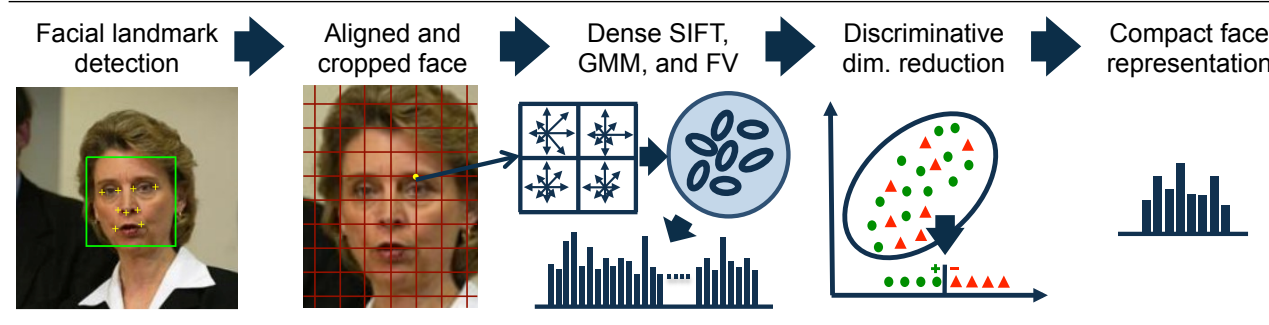

Figure 1: Method overview: a face is encoded in a discriminative compact representation

dimensionality of the descriptor is moderate (e.g. less than 1000 dimensions), so different approaches are required otherwise. One approach is to first reduce the dimensionality generatively, for example by using PCA, and then perform metric learning in a low-dimensional space [ $[\mathbf{0}, \mathbf{⿴}$ ], but this is suboptimal as the first step may lose important discriminative information. Another approach, which we use here, is to optimise directly the projection matrix $W$, as its size depends on the reduced dimensionality, although this results in a non-convex

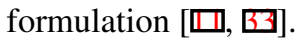

\section{Fisher vector faces representation}

Dense features. The FV construction starts by extracting patch features such as SIFT [ $\square]$ from the image. Rather than sampling locations and scales sparsely by running a carefully tuned face landmark detector, our approach extracts features densely in scale and space. Specifically, $24 \times 24$ pixels patches are sampled with a stride of one pixel and for each patch the root-SIFT representation of [⿴囗口 (referred simply as "SIFT" in the following) is computed. The process is repeated at five scales, with a scaling factors of $\sqrt{2}$. The procedure is run (unless otherwise noted) after cropping and rescaling the face to a $160 \times 125$ image, resulting in about $26 \mathrm{~K} 128$-dimensional descriptors per face. To aggregate these descriptors, the non-linear FV encoding is used, as described briefly below.

Fisher vectors. The FV encoding aggregates a large set of vectors (e.g. the dense SIFT features just extracted) into a high-dimensional vector representation. In general, this is done by fitting a parametric generative model, e.g. the Gaussian Mixture Model (GMM), to the features, and then encoding the derivatives of the log-likelihood of the model with respect to its parameters [ $[\square]$. Following [ $\square]$, we train a GMM with diagonal covariances, and only consider the derivatives with respect to the Gaussian mean and variances. This leads to the representation which captures the average first and second order differences between the (dense) features and each of the GMM centres:

$$
\Phi_{k}^{(1)}=\frac{1}{N \sqrt{w_{k}}} \sum_{p=1}^{N} \alpha_{p}(k)\left(\frac{x_{p}-\mu_{k}}{\sigma_{k}}\right), \quad \Phi_{k}^{(2)}=\frac{1}{N \sqrt{2 w_{k}}} \sum_{p=1}^{N} \alpha_{p}(k)\left(\frac{\left(x_{p}-\mu_{k}\right)^{2}}{\sigma_{k}^{2}}-1\right)
$$

Here, $\left\{w_{k}, \mu_{k}, \sigma_{k}\right\}_{k}$ are the mixture weights, means, and diagonal covariances of the GMM, which is computed on the training set and used for the description of all face images; $\alpha_{p}(k)$ is the soft assignment weight of the $p$-th feature $x_{p}$ to the $k$-th Gaussian. An FV $\phi$ is obtained 
by stacking the differences: $\phi=\left[\Phi_{1}^{(1)}, \Phi_{1}^{(2)}, \ldots, \Phi_{K}^{(1)}, \Phi_{K}^{(2)}\right]$. The encoding describes how the distribution of features of a particular image differs from the distribution fitted to the features of all training images.

To make the dense patch features amenable to the FV description based on the diagonalcovariance GMM, they are first decorrelated by PCA. In our experiments, we applied PCA to SIFT, reducing its dimensionality from 128 to 64 . The FV dimensionality is $2 K d$, where $K$ is the number of Gaussians in the GMM, and $d$ is the dimensionality of the patch feature vector. We note that even though FV dimensionality is high (65536 for $K=512$ and $d=64$ ), it is still significantly lower than the dimensionality of the vector obtained by stacking all dense features (1.7M in our case). Following [四], the performance of an FV is further improved by passing it through signed square-rooting and $L_{2}$ normalisation.

Spatial information. The Fisher vector is an effective encoding of the feature space structure. However, it does not capture the distribution of features in the spatial domain. Several ways of incorporating the spatial information have been proposed in the literature. In [四],

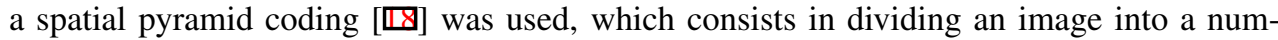
ber of cells and then stacking the FVs computed for each of these cells. The disadvantage of such approach is that the dimensionality of the final image descriptor increases linearly with the number of cells. In [ $[\mathbf{D}]$, a generative model (e.g. GMM) was learnt for the spatial location of each visual word, and FV was used to encode both feature appearance and location. Here we employ a related approach of $[\mathbb{Q}]$, which consists in augmenting the visual features with their spatial coordinates, and then using the FV encoding of the augmented features as the image descriptor. In more detail, our dense features have the following form: $\left[\mathcal{S}_{x y} ; \frac{x}{w}-\frac{1}{2} ; \frac{y}{h}-\frac{1}{2}\right]$, where $\mathcal{S}_{x y}$ is the (PCA-SIFT) descriptor of a patch centred at $(x, y)$, and $w$ and $h$ are the width and height of the face image. The resulting FV dimensionality is thus 67584. Fig. 2 illustrates how Gaussian mixture components are spatially distributed over a face when learnt for a face verification task.

\section{Large-margin dimensionality reduction}

In this section we explain how a high-dimensional FV encoding (Sect. 2) is compressed to a small discriminative representation. The compression is carried out using a linear projection, which serves two purposes: (i) it dramatically reduces the dimensionality of the face descriptors, making them applicable to large-scale datasets; and (ii) it improves the recognition performance by projection onto a subspace with a discriminative Euclidean distance.

In more detail, the aim is to learn a linear projection $W \in \mathbb{R}^{p \times d}, p \ll d$, which projects high-dimensional Fisher vectors $\phi \in \mathbb{R}^{d}$ to low-dimensional vectors $W \phi \in \mathbb{R}^{p}$, such that the squared Euclidean distance $d_{W}^{2}\left(\phi_{i}, \phi_{j}\right)=\left\|W \phi_{i}-W \phi_{j}\right\|_{2}^{2}$ between images $i$ and $j$ is smaller than a learnt threshold $b \in \mathbb{R}$ if $i$ and $j$ are the same person, and larger otherwise. We further impose that these conditions are satisfied with a margin of at least one, resulting in the constraints:

$$
y_{i j}\left(b-d_{W}^{2}\left(\phi_{i}, \phi_{j}\right)\right)>1
$$

where $y_{i j}=1$ iff images $i$ and $j$ contain the faces of the same person, and $y_{i j}=-1$ otherwise.

Note that the Euclidean distance in the $p$-dimensional projected space can be seen as a low-rank Mahalanobis metric in the original $d$-dimensional space:

$$
d_{W}^{2}\left(\phi_{i}, \phi_{j}\right)=\left\|W \phi_{i}-W \phi_{j}\right\|_{2}^{2}=\left(\phi_{i}-\phi_{j}\right)^{T} W^{T} W\left(\phi_{i}-\phi_{j}\right),
$$


where $W^{T} W \in \mathbb{R}^{d \times d}$ is the Mahalanobis matrix defining the metric. Due to the factorisation, the Mahalanobis matrix $W^{T} W$ has rank equal to $p$, i.e. much smaller than the full rank $d$. As a consequence, learning the projection matrix $W$ is the same as learning a low-rank metric $W^{T} W$. Direct optimisation of the Mahalanobis matrix is however quite difficult, as the latter has over 2 billion parameters for the $d=67 \mathrm{~K}$ dimensional FVs. On the contrary, $W$ has $p d=8.5 \mathrm{M}$ parameters for $p=128$, which can be learnt in the large scale learning scenario.

Learning $W$ optimises the following objective function, incorporating the constraints (2) in a hinge-loss formulation:

$$
\arg \min _{W, b} \sum_{i, j} \max \left[1-y_{i j}\left(b-\left(\phi_{i}-\phi_{j}\right)^{T} W^{T} W\left(\phi_{i}-\phi_{j}\right)\right), 0\right]
$$

The minimiser of (4) is found using a stochastic sub-gradient method. At each iteration $t$, the algorithm samples a single pair of face images $(i, j)$ (sampling with equal frequency positive and negative labels $y_{i j}$ ) and performs the following update of the projection matrix:

$$
W_{t+1}= \begin{cases}W_{t} & \text { if } y_{i j}\left(b-d_{W}^{2}\left(\phi_{i}, \phi_{j}\right)\right)>1 \\ W_{t}-\gamma y_{i j} W_{t} \psi_{i j} & \text { otherwise }\end{cases}
$$

where $\psi_{i j}=\left(\phi_{i}-\phi_{j}\right)\left(\phi_{i}-\phi_{j}\right)^{T}$ is the outer product of the difference vectors, and $\gamma$ is a constant learning rate, determined on the validation set. Note that the projection matrix $W_{t}$ is left unchanged if the constraint (2) is not violated, which speed-ups learning (due to the large size of $W$, performing matrix operations at each iteration is costly). We choose not to regularise $W$ explicitly; rather, the algorithm stops after a fixed number of learning iterations (1M in our case).

Finally, note that the objective (4) is not convex in $W$, so initialisation is important. In practice, we initialise $W$ to extract the $p$ largest PCA dimensions. Furthermore, differently from standard PCA, we equalise the magnitude of the dominant eigenvalues (whitening) as the less frequent modes of variation tend to be amongst the most discriminative. It is important to note that PCA-whitening is only used to initialise the learning process, and the learnt metric substantially improves over its initialisation (Sect. 5). In particular, this is not the same as learning a metric on the low-dimensional PCA-whitened data ( $p^{2}$ parameters); instead, a projection $W$ on the original descriptors is learnt ( $p d \gg p^{2}$ parameters), which allows us to fully exploit the available supervision.

\section{Implementation details and extensions}

Face alignment and extraction. Given an image, we first run the Viola Jones detector [B]] to obtain the face detection. Using this detection, we then detect nine facial landmark positions using the publicly available code of [Q]]. Similar to them, we then apply similarity transformation using all these points to transform a face to a canonical frame. In the aligned image, we extract a $160 \times 125$ face region around the landmarks for further processing.

Face descriptor computation. For dense SIFT computation and Fisher vector encoding, we utilised publicly available packages [ $[$, B B] $]$. Dimensionality reduction learning is implemented in MATLAB and takes a few hours to compute on a single core (for each split). Given an aligned and cropped face image, our mexified MATLAB implementation takes $0.6 \mathrm{~s}$ to compute a descriptor on a single CPU core (in the case of 2 pixel SIFT density). 
Diagonal "metric" learning. Apart from the low-rank Mahalanobis metric learning (Sect. 3), we also consider diagonal metric learning on the full-dimensional Fisher vectors. It is carried out using a conventional linear SVM formulation, where features are the vectors of squared differences between the corresponding components of the two compared FVs. We did not observe any improvement by enforcing the positivity of the learnt weights, so it was omitted in practice (i.e. the learnt function is not strictly a metric).

Joint metric-similarity learning. Recently, a "joint Bayesian" approach to face similarity learning has been employed in [Q, 日]. It effectively corresponds to joint learning of a lowrank Mahalanobis distance $\left(\phi_{i}-\phi_{j}\right)^{T} W^{T} W\left(\phi_{i}-\phi_{j}\right)$ and a low-rank kernel (inner product) $\phi_{i}^{T} V^{T} V \phi_{j}$ between face descriptors $\phi_{i}, \phi_{j}$. Then, the difference between the distance and the inner product can be used as a score function for face verification. We consider it as another option for comparing face descriptors (apart from the low-rank metric learning and diagonal metric learning), and incorporate joint metric-similarity learning into our largemargin learning formulation (4). In that case, we perform stochastic updates (5) on both low-dimensional projections $W$ and $V$.

Horizontal flipping. Following []], we considered the augmentation of the test set by taking the horizontal reflections of the two compared images, and averaging the distances between the four possible combinations of the original and reflected images.

\section{Experiments}

\subsection{Dataset and evaluation protocol}

Our framework is evaluated on the popular "Labeled Faces in the Wild dataset" (LFW) [ $\square$ ]. The dataset contains 13233 images of 5749 people downloaded from the Web and is considered the de-facto standard benchmark for automatic face verification. For evaluation, the data is divided into 10 disjoint splits, which contain different identities and come with a list of 600 pre-defined image pairs for evaluation (as well as training as explained below). Of these, 300 are "positive" pairs portraying the same person and the remaining 300 are "negative" pairs portraying different people.

We follow the recommended evaluation procedure $[\square]$ and measure the performance of our method by performing a 10 fold cross validation, training the model on 9 splits, and testing it on the remaining split. All aspects of our method that involve learning, including PCA projections for SIFT, Gaussian mixture models, and the discriminative Fisher vector projections, were trained independently for each fold.

Two evaluation measures are considered. The first one is the Receiving Operating Characteristic Equal Error Rate (ROC-EER), i.e. the false positive rate at the ROC operating point where the false positive and false negative rates are equal [ $[$ ] $]$. This measure reflects the quality of the ranking obtained by scoring image pairs and, as such, is independent on the bias learnt in (2). ROC-EER is used to compare the different stages of the proposed framework. In order to allow a direct comparison with published results, however, our final classification performance is also reported in terms of the classification accuracy (percentage of image pairs correctly classified) - in this case the bias is important.

LFW specifies a number of evaluation protocols, two of which are considered here. In the "restricted setting", only the pre-defined image pairs for each of the splits (fixed by the 
LFW creators) can be used for training. Instead, in the "unrestricted setting" one is given the identities of the people within each split and is allowed to form an arbitrary number, in practice much larger, of positive and negative pairs for training.

\subsection{Framework parameters}

First, we explore how the different parameters of the method affect its performance. The experiments were carried out in the unrestricted setting using unaligned LFW images and a simple alignment procedure described in Sect. 4. We explore the following settings: SIFT density (the step between the centres of two consecutive descriptors), the number of Gaussians in the GMM, the effect of spatial augmentation, dimensionality reduction, distance function, and horizontal flipping. The results of the comparison are given in Table 1. As can be seen, the performance increases with denser sampling and more clusters in the GMM. Spatial augmentation boosts the performance with only a moderate increase in dimensionality (caused by the addition of the $(x, y)$ coordinates to 64-D PCA-SIFT). Our dimensionality reduction to 128 -D achieves 528 -fold compression and further improves the performance. We found that using projection to higher-dimensional spaces (e.g. 256-D) does not improve the performance, which can be caused by overfitting.

As far as the choice of the FV distance function is concerned, a low-rank Mahalanobis metric outperforms both full-rank diagonal metric and unsupervised PCA-whitening, but is somewhat worse than the function obtained by the joint large-margin learning of the Mahalanobis metric and inner product. It should be noted that the latter comes at the cost of slower learning and the necessity to keep two projection matrices instead of one. Finally, using horizontal flipping consistently improves the performance. In terms or the ROC-EER measure, our best result is $93.13 \%$.

\begin{tabular}{|c|c|c|c|c|c|c|}
\hline $\begin{array}{c}\text { SIFT } \\
\text { density }\end{array}$ & $\begin{array}{c}\text { GMM } \\
\text { Size }\end{array}$ & $\begin{array}{c}\text { Spatial } \\
\text { Aug. }\end{array}$ & $\begin{array}{c}\text { Desc. } \\
\text { Dim. }\end{array}$ & $\begin{array}{c}\text { Distance } \\
\text { Function }\end{array}$ & $\begin{array}{c}\text { Hor. } \\
\text { Flip. }\end{array}$ & $\begin{array}{c}\text { ROC- } \\
\text { EER,\% }\end{array}$ \\
\hline 2 pix & 256 & & 32768 & diag. metric & & 89.0 \\
\hline 2 pix & 256 & $\checkmark$ & 33792 & diag. metric & & 89.8 \\
\hline 2 pix & 512 & $\checkmark$ & 67584 & diag. metric & & 90.6 \\
\hline 1 pix & 512 & $\checkmark$ & 67584 & diag. metric & & 90.9 \\
\hline 1 pix & 512 & $\checkmark$ & 128 & low-rank PCA-whitening & & 78.6 \\
\hline 1 pix & 512 & $\checkmark$ & 128 & low-rank Mah. metric & & 91.4 \\
\hline 1 pix & 512 & $\checkmark$ & 256 & low-rank Mah. metric & & 91.0 \\
\hline 1 pix & 512 & $\checkmark$ & 128 & low-rank Mah. metric & $\checkmark$ & 92.0 \\
\hline 1 pix & 512 & $\checkmark$ & $2 \times 128$ & low-rank joint metric-sim. & & 92.2 \\
\hline 1 pix & 512 & $\checkmark$ & $2 \times 128$ & low-rank joint metric-sim. & $\checkmark$ & 93.1 \\
\hline
\end{tabular}

Table 1: Framework parameters: The effect of different FV computation parameters and distance functions on ROC-EER. All experiments done in the unrestricted setting.

\subsection{Learnt projection model visualisation}

Here we demonstrate that the learnt model can indeed capture face-specific features. To visualise the projection matrix $W$, we make use of the fact that each GMM component corresponds to a part of the Fisher vector and, in turn, to a group of columns in $W$. This makes it possible to evaluate how important certain Gaussians are for comparing human face images 


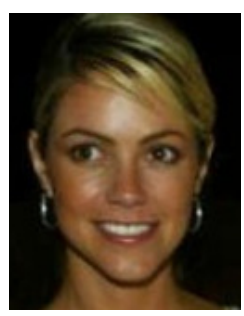

(a)

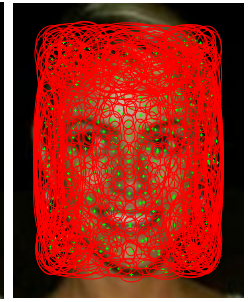

(b)

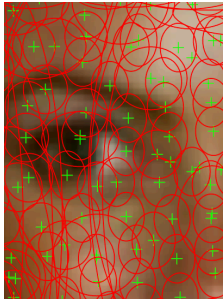

(c)

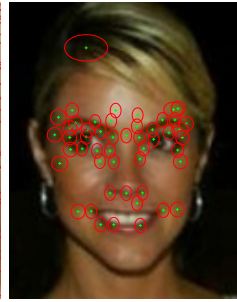

(d)

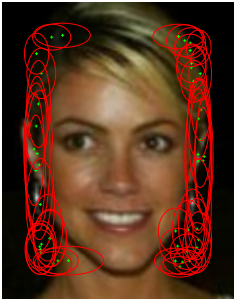

(e)

Figure 2: Coupled with discriminative dimensionality reduction, a Fisher vector can automatically capture the discriminative parts of the face. (a): an aligned face image; (b): unsupervised GMM clusters densely span the face; (c): a close-up of a face part covered by the Gaussians; (d): 50 Gaussians corresponding to the learnt projection matrix columns with the highest energy; (e): 50 Gaussians corresponding to the learnt projection matrix columns with the lowest energy.

by computing the energy (Euclidean norm) of the corresponding column group. In Fig. 2 we show the GMM components which correspond to the groups of columns with the highest and lowest energy. Each Gaussian captures joint appearance-location statistics (Sect. 2), but here we only visualise the location as an ellipse with the centre and radii set to the mean and variances of the spatial components. As can be seen from Fig. 2-d, the 50 Gaussians corresponding to the columns with the highest energy match the facial features without being explicitly trained to do so. They have small spatial variances and are finely localised on the image plane. On the contrary, Fig. 2-e shows how the 50 Gaussians corresponding to the columns with the lowest energy cover the background areas. These clusters are deemed as the least meaningful by our projection learning; note that their spatial variances are large.

\subsection{Comparison with the state-of-the-art}

Unrestricted setting. In this scenario, we compare against the best published results obtained using both single (Table 2, left-bottom) and multi-descriptor representations (Table 2, left-top). Similarly to the previous section, the experiments were carried out using unaligned LFW images, processed as described in Sect. 4. This means that the outside training data is only utilised in the form of a simple landmark detector, trained by [ $[\boldsymbol{\theta}]$.

Our method achieves $93.03 \%$ face verification accuracy, closely matching the state-ofthe-art method of [ $\mathbf{\theta}]$, which achieves $93.18 \%$ using LBP features sampled around 27 landmarks. It should be noted that (i) the best result of [日] using SIFT descriptors is $91.77 \%$; (ii) we do not rely on multiple landmark detection, but sample the features densely. The ROC curves of our method as well as the other methods are shown in Fig. 3.

Restricted setting. In this strict setting, no outside training data is used, even for the landmark detection. Following [四], we used centred $150 \times 150$ crops of "LFW-funneled" images, provided as a part of the LFW dataset. We found that the limited amount of training data, available in this setting, is insufficient for dimensionality reduction learning. Therefore, we learnt a diagonal "metric" function using an SVM as described in Sect. 4. Achieving the verification accuracy of $87.47 \%$, our descriptor sets a new state-of-the-art in the restricted setting (Table 2, right), outperforming the recently published result of [四] by $3.4 \%$. It 

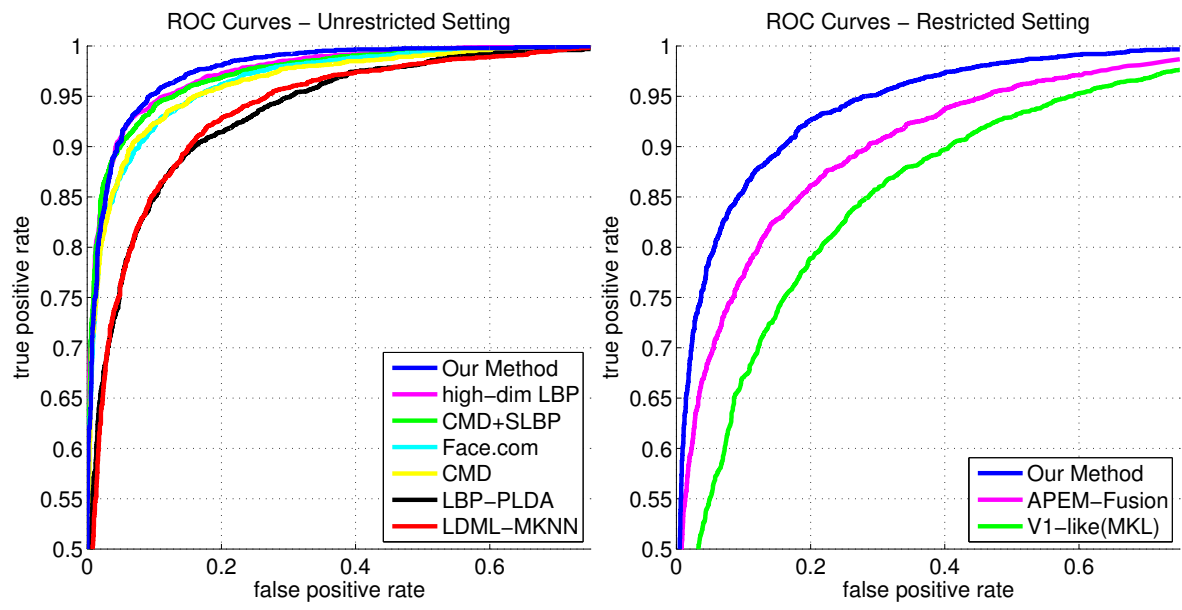

Figure 3: Comparison with the state-of-the-art: ROC curves of our method and the stateof-the-art techniques in LFW-unrestricted (left) and LFW-restricted (right) settings.

should be noted that while [] also use GMMs for dense feature clustering, they do not utilise the compressed Fisher vector encoding, but keep all extracted features for matching, which imposes a limitation on the number of features that can be extracted and stored. In our case, we are free from this limitation, since the dimensionality of an FV does not depend on the number of features it encodes. The best result of [ष] was obtained using two types of features and GMM adaptation ("APEM Fusion"). When using non-adapted GMMs (as we do) and SIFT descriptors ("PEM SIFT"), their result is $6 \%$ worse than ours.

Our results in both unrestricted and restricted settings confirm that the proposed face descriptor can be used in both small-scale and large-scale learning scenarios, and is robust with respect to the face alignment and cropping technique.

\section{Conclusion}

In this paper, we have shown that an off-the-shelf image representation based on dense SIFT features and Fisher vector encoding achieves state-of-the-art performance on the challenging "Labeled Faces in the Wild" dataset. The use of dense features allowed us to avoid applying a large number of sophisticated face landmark detectors. Also, we have presented a largemargin dimensionality reduction framework, well suited for high-dimensional Fisher vector representations. As a result, we obtain an effective and efficient face descriptor computation pipeline, which can be readily applied to large-scale face image repositories.

It should be noted that the proposed system is based upon a single feature type. In our future work, we are planning to investigate multi-feature image representations, which can be readily incorporated into our framework.

\section{Acknowledgements}

This work was supported by ERC grant VisRec no. 228180 and EU Project AXES ICT269980. 


\begin{tabular}{|c|c|c|c|}
\hline \multicolumn{2}{|c|}{ Unrestricted setting } & \multicolumn{2}{|c|}{ Restricted setting } \\
\hline Method & Mean Acc. & Method & Mean Acc. \\
\hline LDML-MkNN [ா]] & $0.8750 \pm 0.0040$ & V1-like/MKL [四] & $0.7935 \pm 0.0055$ \\
\hline Combined multishot [B] & $0.8950 \pm 0.0051$ & PEM SIFT [ष्व] & $0.8138 \pm 0.0098$ \\
\hline Combined PLDA [四] & $0.9007 \pm 0.0051$ & APEM Fusion [四] & $0.8408 \pm 0.0120$ \\
\hline face.com [B]] & $0.9130 \pm 0.0030$ & Our Method & $0.8747 \pm \mathbf{0 . 0 1 4 9}$ \\
\hline CMD + SLBP [Ш] & $0.9258 \pm 0.0136$ & & \\
\hline LBP multishot [B] & $0.8517 \pm 0.0061$ & & \\
\hline LBP PLDA [四] & $0.8733 \pm 0.0055$ & & \\
\hline SLBP [ם] & $0.9000 \pm 0.0133$ & & \\
\hline CMD [ㅁ] & $0.9170 \pm 0.0110$ & & \\
\hline High-dim SIFT [田] & $0.9177 \pm \mathrm{N} / \mathrm{A}$ & & \\
\hline High-dim LBP [可] & $0.9318 \pm 0.0107$ & & \\
\hline Our Method & $0.9303 \pm 0.0105$ & & \\
\hline
\end{tabular}

Table 2: Left: Face verification accuracy in the unrestricted setting. Using a single type of local features, our method achieves the new state-of-the-art among the non-commercial methods, and is competitive compared to the best commercial systems. Right: Face verification accuracy in the restricted setting (no outside training data). Our method achieves the new state-of-the-art in this strict setting.

\section{References}

[1] R. Arandjelović and A. Zisserman. Three things everyone should know to improve object retrieval. In Proc. CVPR, 2012.

[2] P. Belhumeur, J. Hespanha, and D. Kriegman. Eigenfaces vs. Fisherfaces: Recognition using class specific linear projection. IEEE PAMI, 19(7):711-720, 1997.

[3] T. Berg and P. N. Belhumeur. Tom-vs-Pete classifiers and identity-preserving alignment for face verification. In Proc. BMVC., 2012.

[4] K. Chatfield, V. Lempitsky, A. Vedaldi, and A. Zisserman. The devil is in the details: an evaluation of recent feature encoding methods. In Proc. BMVC., 2011.

[5] D. Chen, X. Cao, L. Wang, F. Wen, and J. Sun. Bayesian face revisited: A joint formulation. In Proc. ECCV, pages 566-579, 2012.

[6] D. Chen, X. Cao, F. Wen, and Sun J. Blessing of dimisionality: High dimensional feature and its efficient compression for face verification. In Proc. CVPR, 2013.

[7] G. Csurka, C. Bray, C. Dance, and L. Fan. Visual categorization with bags of keypoints. In Workshop on Statistical Learning in Computer Vision, ECCV, pages 1-22, 2004.

[8] M. Dantone, J. Gall, G. Fanelli, and L. van Gool. Real-time facial feature detection using conditional regression forests. In Proc. CVPR, 2012.

[9] M. Everingham, J. Sivic, and A. Zisserman. Taking the bite out of automatic naming of characters in TV video. Image and Vision Computing, 27(5), 2009. 
[10] M. Guillaumin, J. Verbeek, and C. Schmid. Is that you? metric learning approaches for face identification. In Proc. ICCV, 2009.

[11] M. Guillaumin, J. Verbeek, and C. Schmid. Multiple instance metric learning from automatically labeled bags of faces. In Proc. ECCV, pages 634-647, 2010.

[12] C. Huang, S. Zhu, and K. Yu. Large scale strongly supervised ensemble metric learning, with applications to face verification and retrieval. (TR115), 2011.

[13] G. B. Huang, M. Ramesh, T. Berg, and E. Learned-Miller. Labeled faces in the wild: A database for studying face recognition in unconstrained environments. Technical Report 07-49, University of Massachusetts, Amherst, October 2007.

[14] T. Jaakkola and D. Haussler. Exploiting generative models in discriminative classifiers. In NIPS, pages 487-493, 1998.

[15] H. Jégou, M. Douze, C. Schmid, and P. Pérez. Aggregating local descriptors into a compact image representation. In Proc. CVPR, 2010.

[16] J. Krapac, J. Verbeek, and F. Jurie. Modeling spatial layout with fisher vectors for image categorization. In Proc. ICCV, pages 1487-1494, 2011.

[17] N. Kumar, A. C. Berg, P. Belhumeur, and S. K. Nayar. Attribute and simile classifiers for face verification. In Proc. ICCV, 2009.

[18] S. Lazebnik, C. Schmid, and J Ponce. Beyond Bags of Features: Spatial Pyramid Matching for Recognizing Natural Scene Categories. In Proc. CVPR, 2006.

[19] H. Li, G. Hua, J. Brandt, and J. Yang. Probabilistic elastic matching for pose variant face verification. In Proc. CVPR, 2013.

[20] P. Li, Y. Fu, U. Mohammed, J. H. Elder, and S. J. D. Prince. Probabilistic models for inference about identity. IEEE PAMI, 34(1):144-157, Nov 2012.

[21] D. Lowe. Distinctive image features from scale-invariant keypoints. IJCV, 60(2):91110, 2004.

[22] H. V. Nguyen and L. Bai. Cosine similarity metric learning for face verification. In Proc. Asian Conf. on Computer Vision, 2010.

[23] E. Nowak, F. Jurie, and B. Triggs. Sampling strategies for bag-of-features image classification. In Proc. ECCV, 2006.

[24] F. Perronnin, J. Sánchez, and T. Mensink. Improving the Fisher kernel for large-scale image classification. In Proc. ECCV, 2010.

[25] N. Pinto and D. Cox. Beyond simple features: A large-scale feature search approach to unconstrained face recognition. In Proc. Int. Conf. Autom. Face and Gesture Recog., 2011.

[26] N. Pinto, J. J. DiCarlo, and D. D. Cox. How far can you get with a modern face recognition test set using only simple features? In Proc. CVPR, 2009. 
[27] J. Sánchez and F. Perronnin. High-dimensional signature compression for large-scale image classification. In Proc. CVPR, 2011.

[28] J. Sánchez, F. Perronnin, and T. Emídio de Campos. Modeling the spatial layout of images beyond spatial pyramids. Pattern Recognition Letters, 33(16):2216-2223, 2012.

[29] K. Simonyan, A. Vedaldi, and A. Zisserman. Descriptor learning using convex optimisation. In Proc. ECCV, 2012.

[30] J. Sivic and A. Zisserman. Video Google: A text retrieval approach to object matching in videos. In Proc. ICCV, volume 2, pages 1470-1477, 2003.

[31] Y. Taigman and L. Wolf. Leveraging billions of faces to overcome performance barriers in unconstrained face recognition. 2011.

[32] Y. Taigman, L. Wolf, and T. Hassner. Multiple one-shots for utilizing class label information. In Proc. BMVC., 2009.

[33] L. Torresani and K. Lee. Large margin component analysis. In NIPS, pages 1385-1392. MIT Press, 2007.

[34] A. Vedaldi and B. Fulkerson. VLFeat - an open and portable library of computer vision algorithms. In ACM Multimedia, 2010.

[35] P. Viola and M. Jones. Robust real-time object detection. In IJCV, volume 1, 2001.

[36] K.Q. Weinberger, J. Blitzer, and L. Saul. Distance metric learning for large margin nearest neighbor classification. In Y. Weiss, B. Schölkopf, and J. Platt, editors, NIPS. MIT Press, 2006.

[37] L. Wolf, T. Hassner, and Y. Taigman. Descriptor based methods in the wild. In Faces in Real-Life Images Workshop in European Conference on Computer Vision, 2008.

[38] L. Wolf, T. Hassner, and Y. Taigman. Similarity scores based on background samples. In Proc. Asian Conf. on Computer Vision, 2009.

[39] Q. Yin, X. Tang, and Sun J. Face recognition with learning-based descriptor. In Proc. CVPR, 2011.

[40] Y. Ying and P. Li. Distance metric learning with eigenvalue optimization. J. Machine Learning Research, 2012.

[41] X. Zhou, K. Yu, T. Zhang, and T. S. Huang. Image classification using super-vector coding of local image descriptors. In Proc. ECCV, 2010.

[42] X. Zhu and D. Ramanan. Face detection, pose estimation and landmark localization in the wild. In Proc. CVPR, 2012. 